\title{
A CMOS CHARGE SENSITIVE AMPLIFIER FOR SILICON STRIP DETECTORS: DESIGN CRITERIA AND TEST RESULTS
}

\author{
A. Olsen \\ Center for Industrial Research, Oslo, Norway \\ T. Buran, K. Eilertsen, G. Maehlum, A. Read, B. Skaali and I. Spydevold \\ University of Oslo, Oslo, Norway
}

\section{ABSTRACT}

A low noise charge sensitive amplifier has been developed with a commercial CMOS process. We present measurements of the amplifier's performance when used to read out a silicon strip detector exposed to a particle beam. A few protopypes have been exposed to large doses of radiation in order to study the radiation hardness.

Presented to the London Conference on position-sensitive detectors 7-11 September 1987 


\section{TABLE OF CONTENTS}

Page

1. INTRODUCTION 3

2. AMPLIFIER DESIGN 3

3. EXPERIMENTAL SET-UP FOR BEAM TESTS 5

4. AMPLIFIER PERFORMANCE IN THE BEAM 6

5. COMPARISON WITH OTHER CHARGE AMPLIFIERS 7

6. RADIATION HARDNESS TESTS 8

$\begin{array}{ll}\text { REFERENCES } & 10\end{array}$ 


\section{INTRODUCTION}

The use of silicon microstrip detectors for precise measurements of charged particle trajectories in fixed target experiments is now an established technique [1]. In these experiments the detectors have been read out with hybrid front-end electronics. The presence of large area silicon detectors in collider experiments compels the use of VLSI front-end electronics due to the large number of readout channels and space limitations. There is at present no collider experiment utilizing silicon strip or microstrip detectors with VLSI readout.

At the Group for Experimental High Energy Physics at the University of Oslo a track detector composed of silicon chips is being constructed for the Small Angle Tagger (SAT) for the DELPHI Experiment at LEP [2]. The detector will consist of 6 planes, 3 planes in each direction from the collision region. The planes are constructed from $\sim 2000$ detector chips each having 40 to 50 strips. Every silicon strip will be read out by one channel of a VLSI circuit that will Amplify the charge signal, Discriminate against noise, and Encode (ADE [3]) the hit pattern of strips into digital words. Each channel contains also a capacitor at the input to filter away DC noise. The VLSI chip contains 64 channels in an area of $6 \times 7 \mathrm{~mm}$. Both the detectors and the VLSI circuits have been developed at the Center for Industrial Research in Oslo [4]. In this report we present measurements of the performance of a prototype of the charge amplifier to be used in the ADE circuit.

\section{AMPLIFIER DESIGN}

Monolithic integration is the only approach able to yield the required number of channels in the available space at an acceptable cost per channel. The circuits were designed in CMOS due to the large integration scale and the desire to combine digital and analog functions on the same chip. The high level of low frequency noise characteristic of CMOS analog circuits was expected to be minimized by careful design. The prototype amplifiers were produced by the Austrian firm AMI [5] as part of a Nordic Multichip Project. The process used has a linewidth of $5 \mu$ and the thickness of the gate oxide is $800 \mathrm{~A}$.

The amplifier consists of three complementary inverters working as push-pull stages as shown in fig. 1. Complementary inverters are used because of their noise performance and simple structure. It is easily shown that the equivalent input noise of such an inverter is given by 


$$
\frac{1}{2}\left(e_{1}^{2}+e_{2}^{2}\right)^{1 / 2}
$$

where $e_{1}$ and $e_{2}$ are the individual equivalent input noises for the two transistors. The equivalent input noise in these stages can be restricted to the input transistor. The amplifier is an integrator with a feedback capacitor of $0.2 \mathrm{pF}$. The chip layout was done with a double polysilicon layer process since polysilicon layers form linear capacitors with relatively high capacitance. The prototype amplifier used in the measurements was connected to the output bonding pad through a buffer and the chip was packed in a dual-in-line chip carrier.

The integrator is reset by a transistor switch shunting the capacitor. The switch has two half size dummy transistors on each side which collect the transistor channel charge ejected by the switch transistor when the reset signal is applied. The amplifier is stabilized at the quiescent point by DC feedback when in reset mode. In active mode there is no such feedback and the amplifier is drifting. This implies that the amplifier should be reset periodically. The amplifier is not expected to function properly if the active period is longer than $1 \mathrm{~ms}$ or if the reset period is shorter than 1 us. These periods are defined by an externally applied signal which is high (low) during reset mode (active mode). High and low are "TTL" levels: $+5 \mathrm{~V}$ and $0 \mathrm{~V}$ respectively.

The primary noise mechanisms in a MOS transistor are the charge carriers' thermal motion and their interactions with electron states in the gate oxide near the interface and in the depletion layer. These effects produce white noise and $1 / f$ noise. The total equivalent transistor input noise is proportional to

$$
\left(\frac{1}{f W L}+\frac{2 L}{3 W \sqrt{ }\left(I_{d}\right)}\right)^{1 / 2}
$$

where $f$ is the frequency, $I_{d}$ the quiescent current, and $W$ and $L$ are respectively the width and length of the transistor channel. This expression shows that the noise is inversely proportional to the quiescent current. The gains of the inverter stages have the same behaviour. In the tradeoff between gain and noise, low noise is given priority. Three inverting stages are therefore necessary to achieve sufficient gain and negative feedback. When DC feedback is used to control the quiescent point, it is important to maintain approximately the same DC level throughout the three stages during the reset period and it is important to keep the transfer characteristics of all stages equal. If either of these conditions is not met, one of the stages may go into saturation, destroying the feedback effect and introducing distortions. In order to match the last two stages to the first, which is designed for low noise, their 
transistors must be relatively wide. This puts constraints on the minimum current in the last two stages. The total DC current is approximately $0.9 \mathrm{~mA}$ and the output resistance is calculated to be $600 \mathrm{k} \Omega$.

Frequency compensation is necessary in the amplifier. This is done by the pole splitting network in the last stage. A simulation of the circuit predicts a phase margin of $>60^{\circ}$ and a unity gain frequency of nearly $10 \mathrm{MHz}$.

\section{EXPERIMENTAL SET-UP FOR BEAM TESTS}

Seven silicon strip detectors were aligned in the beam, as shown schematically in fig. 2. The detectors were mounted on printed circuit boards (PCB's) and bonded directly from the aluminium covered detector strips to gold plated conductors on a PCB. The PCB's were then mounted on supports attached to a high precision optical bench. Plane $\times 2$ could be moved in the $x$ direction by means of a micrometer screw.

The silicon detectors were samples from a prototype batch of detectors produced by SI [6] with a geometry suitable for the DELPHI luminosity monitor. The production process involves deposition and subsequent diffusion of the impurity atoms rather than the technique of implantation. The strip pitch of the detectors is $1 \mathrm{~mm}$ with $0.9 \mathrm{~mm}$ strip width. The geometry corresponds to a $5^{\circ}$ sector of a circle with a radius at the first strip of $10 \mathrm{~cm}$.

The CMOS amplifier was connected to one strip of the detector in plane $\times 2$ through $15 \mathrm{~cm}$ of cable. The detector capacitance is $\sim 10 \mathrm{pF}$, the cable capacitance $7.5 \mathrm{pF}$, and other stray capacitances were estimated to be 2-3 pF. The total input capacitance was therefore $\sim 20 \mathrm{pF}$. This capacitance is somewhat larger than what is the case when the strips are directly bonded to the VLSI circuit. Since the output stage of the amplifier was not able to drive the $20 \mathrm{~m}$ of coaxial cable between the experimental area and the counting barrack, a simple cable driving circuit was designed (based on the LF 356 operational amplifier). The LF 356 also performed the DC level shift necessary to form the negative signals required by the ADC's (LeCroy 2249A).

The strips of the other detector planes were connected to hybrid charge amplifiers supplied by Aurel Microsystem [7]. The hybrid amplifiers were mounted on plug-in PCB's located beside the optical bench. The amplifier electronics and strip 
detector planes were placed inside a light-tight copper box for electromagnetic shielding. Between the hybrid amplifiers and the ADC's in the counting barrack there were $20 \mathrm{~m}$ of twisted pair cables, line receivers, fast amplifiers, and $100 \mathrm{~ns}$ of delay. Some of the signals were fanned out before the $100 \mathrm{~ns}$ and used in the trigger.

The response of the CMOS amplifier to the charge produced when a minimum ionizing particle traverses $300 \mu \mathrm{m}$ of silicon is a negative voltage step of $\sim 10 \mathrm{mV}$ (fig. 3). The gains of the four amplifiers (each on its own chip) available for testing were measured by injecting a known test charge and found to be within $10 \%$ of each other. The ADC's used in the tests were of the charge sensitive type (current integrating). The voltage step was digitized by splitting the signal, delaying one of the split signals, and feeding the delayed and undelayed signals to two different ADC modules. The delayed signal samples the amplifier output immediately "before" the passage of a beam particle. This electronics chain serves as a band-pass filter with the cut of frequencies given by the integration time ( $A D C$ gate length) and the time between the two integrations. This way of reading out the amplifier is comparable to the way the ADE chip will function.

The reset pulses necessary for the operation of the amplifier were obtained from a free running pulse generator and were typically several microseconds long. It was found to be necessary to inhibit the trigger for several microseconds after the end of a reset pulse due to false triggers caused by noise introduced on the hybrid amplifiers by the reset pulse.

The trigger consisted of a coincidence of signals from two scintillators and signals from the detector planes. The scintillators (denoted S1 and S2 in fig. 2) were located up- and down-stream of the optical bench supporting the detector planes and had a cross sectional area of $10 \times 10 \mathrm{~mm}$. The trigger signal from the detector planes consisted of a coincidence of the OR'ed signals from four adjacent strips in detector planes $X 1, Y 1, \times 5$ and $Y 2$.

\section{AMPLIFIER PERFORMANCE IN THE BEAM}

Fig. 3 is an oscilloscope picture of a typical response to a minumum ionizing particle passing through the detector. 
The pulse height distribution from the amplifier connected to a detector strip exposed to minimum ionizing particles is shown in fig. 4. Figs $4(a)$ and $4(b)$ show the pulse height distributions of the samples taken before and after the passage of beam particles and fig. 4(c) shows the event-by-event difference of the two spectra (an arbitrary constant of 200 ADC counts has been added to the difference). The expected Landau distribution is separated clearly from the noise pedestal.

The signal to noise ratio $(S / N)$ is defined as the difference between the positions of the Landau and noise peaks divided by the Root-Mean-Square (RMS) of the noise distribution. The position of the Landau peak, which corresponds to the most probable energy deposition, is obtained from fits to the ADC data of a truncated Landau distribution convoluted with a Gaussian resolution. The free parameters of the fit were the normalization, the most probable energy deposition, and the "width" of the Landau distribution [8]. The standard deviation of the Gaussian resolution was that obtained from the pedestal distribution.

The signal to noise ratio is expected to fall as $\Delta t$, the time between the two sampling $A D C$ gates, increases due to the high level of low frequency noise. The observed dependence of $S / N$ on $\Delta t$ is summarized in table 1 . The drop in the $S / N$ ratio when $\Delta t$ gets short is due to the fact that the two samplings get too closely spaced in time compared to the fall time of the voltage step.

The optimum gate length is determined by the frequency spectrum of the noise and the overall gain desired. A short gate filters low frequency noise and reduces gain while a long gate filters high frequency noise and increases gain. The observed dependence of the $\mathrm{S} / \mathrm{N}$ ratio on the gate length is summarized in table 2 . The drop in the $\mathrm{S} / \mathrm{N}$ ratio for a gate length of $400 \mathrm{~ns}$ occurs because the first sample covers the beginning of the voltage step.

The signal to noise ratio is not expected to depend on the length of the reset pulse as long as the amplifier is completely reset. This is roughly confirmed by the results listed in table 3.

\section{COMPARISON WITH OTHER CHARGE AMPLIFIERS}

The signal to noise ratio of the CMOS amplifier was observed to be comparable to that of the hybrid amplifiers used in the test set-up [7]. 
The signal to noise ratios reported here are somewhat lower than those obtained with the Microplex chips [9]. Some of them can be attributed to differences between the two test set-ups. The Microplex chip was directly bonded to the microstrip detector while for the present measurements the amplifier was connected to the detector through a $15 \mathrm{~cm}$ long cable. It is estimated that stray capacitances added $10 \mathrm{pF}$ to the detector capacitance (of $10 \mathrm{pF}$ ). Since the collected charge is largely dependent on the input capacitance, the signal to noise ratio is expected to increase when the amplifier chip is bonded directly to the detector.

\section{RADIATION HARDNESS TESTS}

The radiation tests were done at the Gamma Irradiation Plant at The Institute of Energy Technology at Kjeller outside Oslo.

Three of the prototype preamplifiers were exposed to radiations from a $60 \mathrm{~K}$ Curie $\mathrm{Co}^{60}$ source.

The chips were placed at a calibrated position with respect to the source. The exposure times were in steps of $30^{\prime \prime}$ to several minutes. The chips were not powered during exposure. Fig. 5(a) shows the behaviour of the standard deviation of the output signal as the radiation dose is increased. The input signal was a test pulse equivalent to approximately two minumum ionizing particles. As explained above the output signal is taken as the difference between two samplings, before and after the test pulse injection respectively. Fig. 5(b) shows the gain's behaviour as a function of the radiation dose. The gain is normalized to one at zero radiation.

In both figs 5(a) and 5(b) we observe that at a radiation dose of approximately $8 \mathrm{Krad}$ the chip starts to malfunction.

The three chips behaved very similar and the figures show the average behaviour. 


\section{Acknowledgements}

The authors are grateful to B. Bjeld and S.I. Christiansen for building the mechanical part of the test set-up and to J.B. Johansen for laying out the printed circuit boards and producing the driver electronics. We also thank J. Wikne for building the fast amplifiers. We thank Dr. Nils Kaltenbran for assistance during the radiation tests. This work was supported in part by the Norwegian Research Council for Science and the Humanities. 


\section{REFERENCES}

[1] B. Hyams et al., Nucl. Instr. and Meth. 205 (1983) 99;

G. Bellini et al., Nucl. Instr. and Meth. 196 (1982) 351.

[2] L. Bugge et al., University of Oslo, Report 86-10 (1986).

[3] E. Nygard, Center of Industrial Research, Report 85 405-2 (1985).

[4] Center for Industrial Research, Oslo, Norway.

[5] AMI Microsystems Ltd., Unter Premstätten, Austria.

[6] SI, Center for Industrial Research, Oslo, Norway.

[7] Aurel Microsystems, Tradate, Italy, The development of the amplifier is described in detail in J.P. Avondo et al., Nucl. Instr. and Meth. A24l (1985) 107.

[8] F. Hancock et al., Phys. Rev. A28 (1983) 615.

[9] G. Anzivino et al., Nucl. Instr. and Meth. A243 (1986) 153;

G. Anzivino et al., CERN Internal Note, CERN-EP/RH-cp-176N. 
Table 1: The signal to noise ratio as a function of $\Delta t$, the time between the two ADC gates (for $T_{G}=250 \mathrm{~ns}, T_{R}=4 \mu \mathrm{s}$ ).

\begin{tabular}{|c|c|}
\hline$\Delta \mathrm{t}(\mu \mathrm{s})$ & Signal/noise (RMS units) \\
\hline 3.0 & $12.0 \pm 0.4$ \\
2.5 & $12.4 \pm 0.4$ \\
2.0 & $12.5 \pm 0.4$ \\
1.5 & $10.6 \pm 0.3$ \\
1.0 & $10.7 \pm 0.4$ \\
\hline
\end{tabular}

Table 2: The signal to noise ratio as a function of $T_{G}$ the $A D C$ gate length (for $\Delta t=2 \mu s, T_{R}=4 \mu s$ ).

\begin{tabular}{|c|c|}
\hline$T_{G}(n s)$ & Signal/noise (RMS units) \\
\hline 200 & $11.2 \pm 0.4$ \\
300 & $12.6 \pm 0.4$ \\
350 & $11.5 \pm 0.4$ \\
400 & $10.6 \pm 0.3$ \\
\hline
\end{tabular}

Table 3: The signal to noise ratio as a function of $T_{R}$ the length of the reset pulse (for $\Delta t=2 \mu s, T_{G}=250 \mathrm{~ns}$ ).

\begin{tabular}{|c|c|}
\hline$T_{R}(\mu s)$ & Signal/noise (RMS units) \\
\hline 0.5 & $12.6 \pm 0.4$ \\
1.0 & $11.4 \pm 0.3$ \\
1.5 & $11.5 \pm 0.3$ \\
2.0 & $12.8 \pm 0.3$ \\
2.5 & $13.0 \pm 0.3$ \\
3.0 & $13.4 \pm 0.3$ \\
3.5 & $12.9 \pm 0.3$ \\
\hline
\end{tabular}




\section{FIGURE CAPTIONS}

Fig. 1 Circuit diagram of the prototype amplifier.

Fig. 2 The beam set-up: $S 1$ and $S 2$ are scintillators, $Y 1$ and $Y 2$ are strip detectors with their strips aligned horizontally, and $\times 1-\times 5$ are strip detectors with their strips aligned vertically. The CMOS-VLSI amplifier read out the centre strip of detector $X 2$.

Fig. 3 An oscilloscope picture of a preamplifier's response to a minimum ionizing particle interacting in the detector. The vertical scales are $10 \mathrm{mV} / \mathrm{div}$ and $0.5 \mathrm{~V} / \mathrm{div}$ for the signal and the gates respectively. The horizontal scale is $500 \mathrm{~ns} / \mathrm{div}$.

Fig. 4 The pulse height distribution for the CMOS-VLSI amplifier connected to a detector strip exposed to minimum ionizing particles:
(a) sample taken before the arrival of a beam particle,
(b) sample taken after the arrival of a beam particle,
(c) event-by-event difference of the two samples.

Fig. 5 (a) The $\sigma$ of the response to the test pulses as a function of the radiation dose.

(b) The gain of the preamplifier as a function of the radiation dose. The gain is normalized to one at zero dose. 


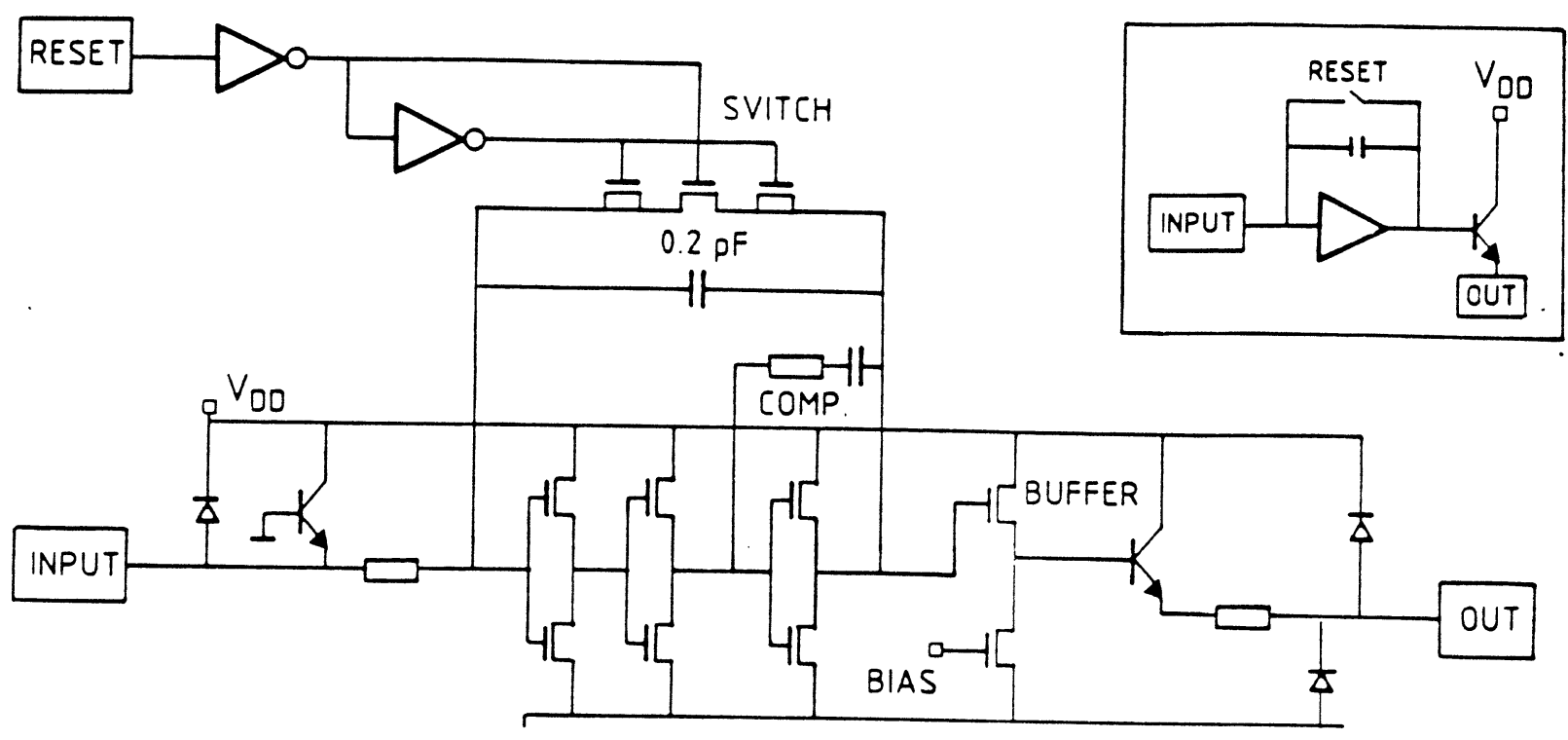

Fig. 1 


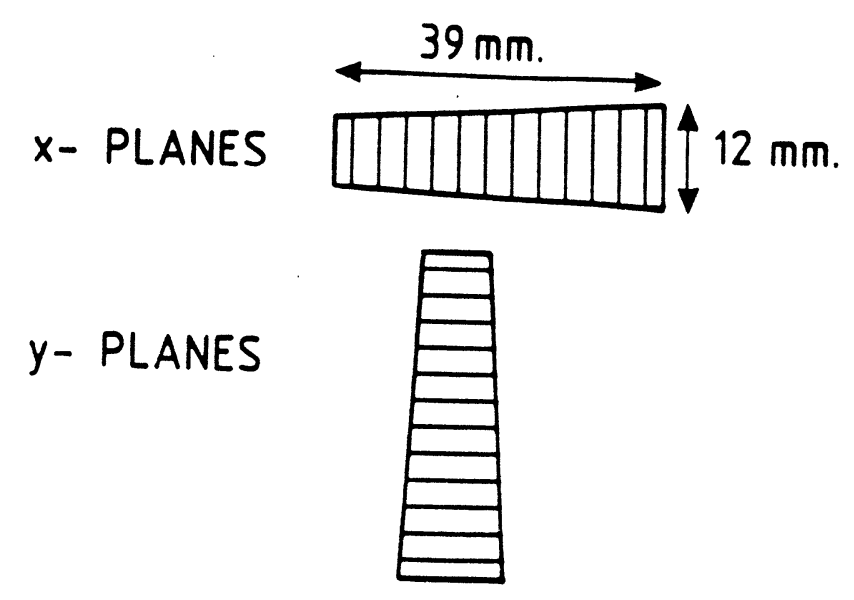

DISTANCES ALONG THE BEAM (THROUGH CENTER OF ALL DETECTORS)
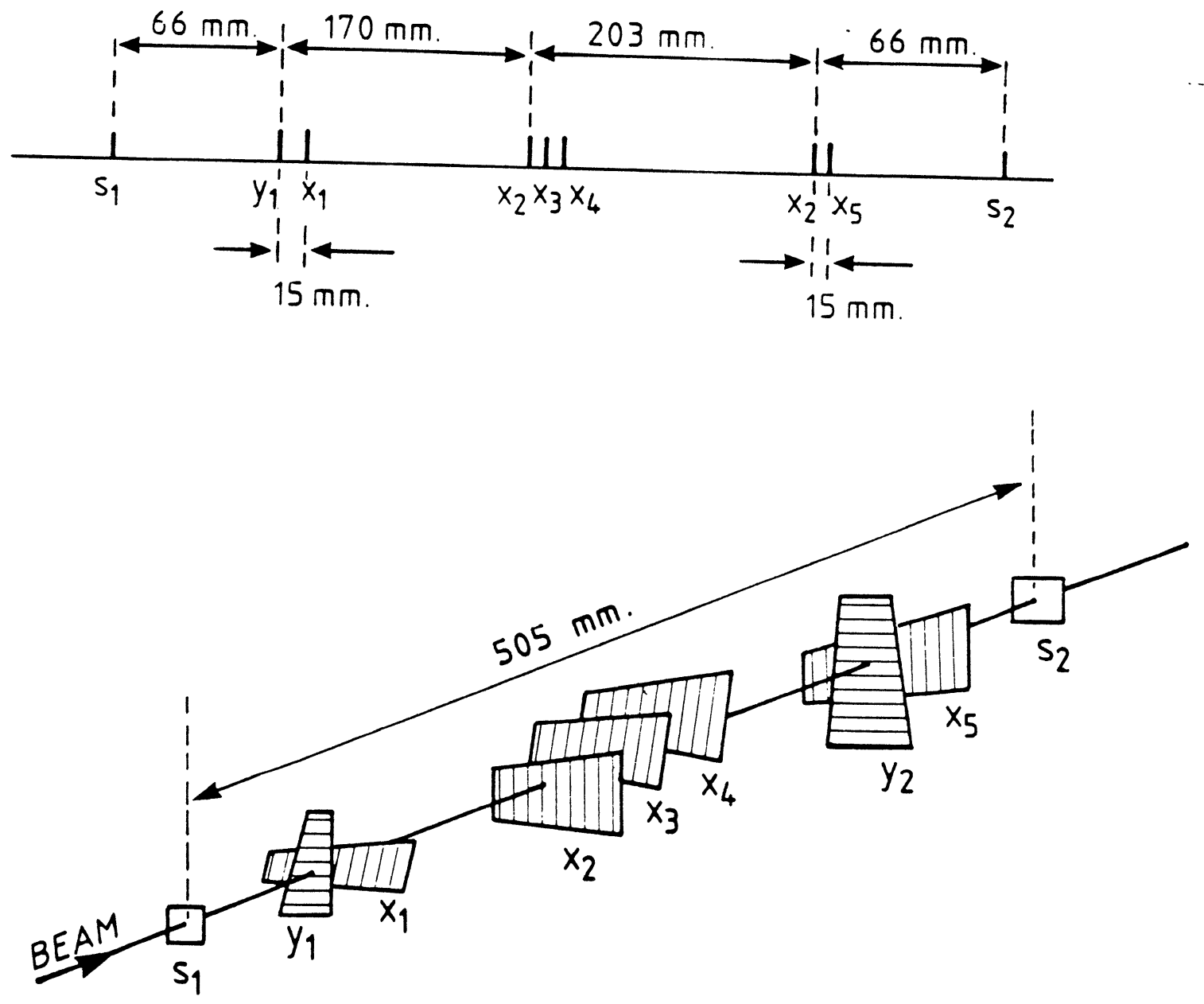

Fig. 2 


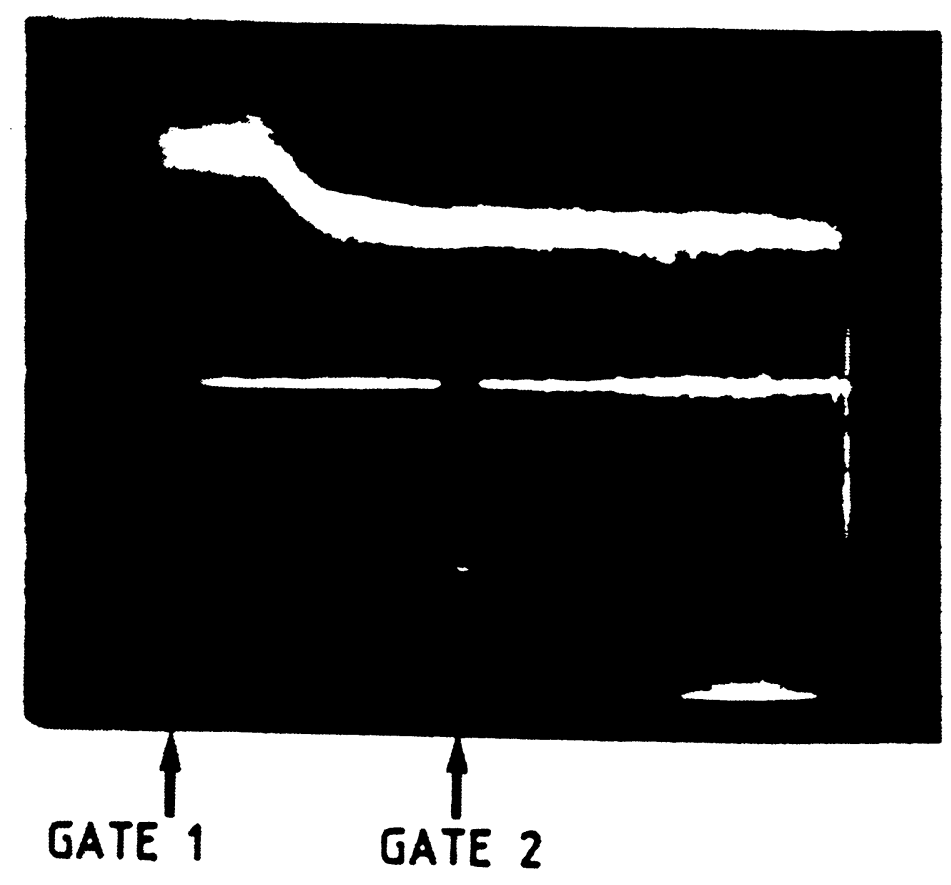

Eig. 3

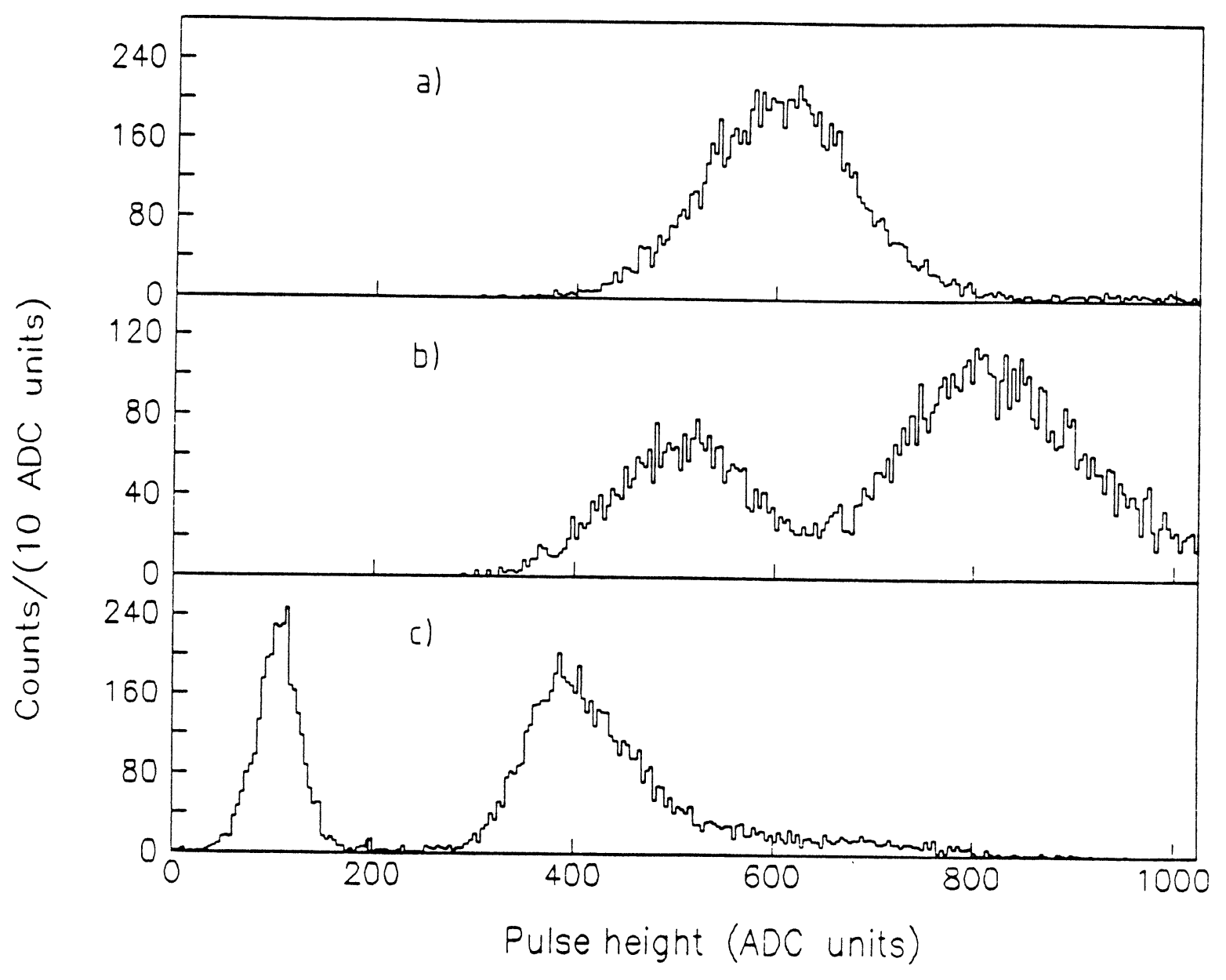

Fig. 4 


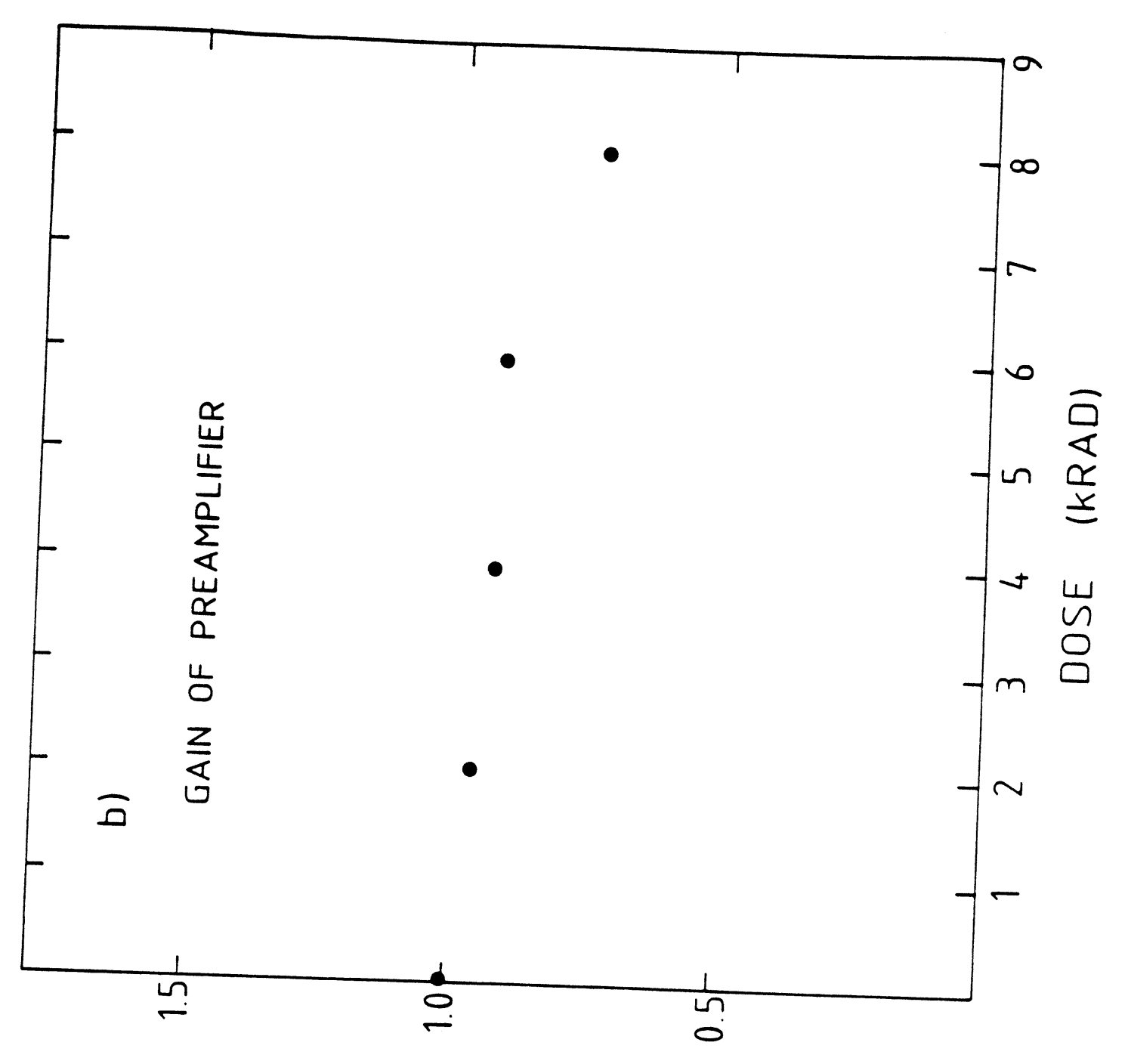

in

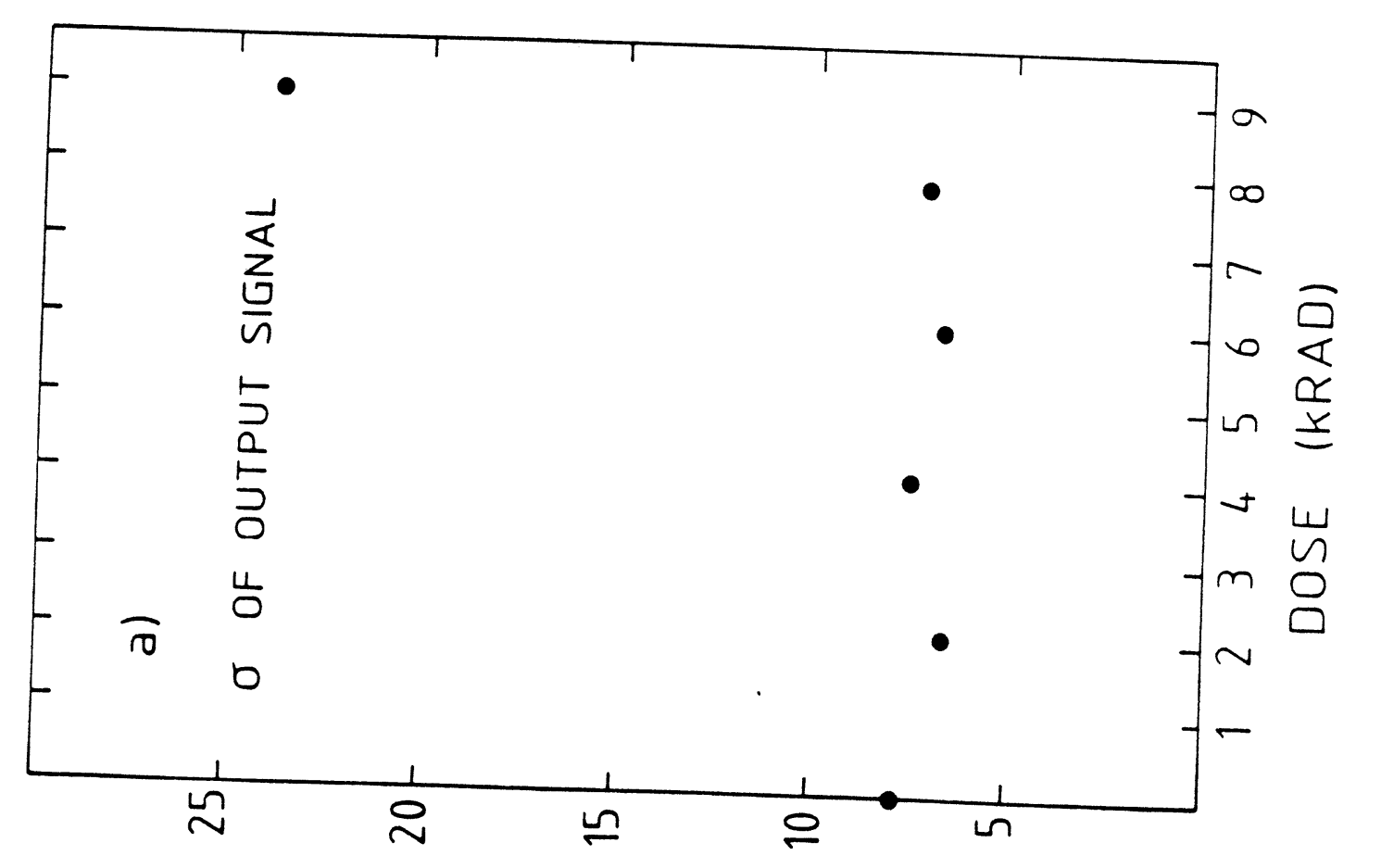

